\title{
The African-American population with a low allele frequency of SNP rs1990760 (T allele) in IFIH1 predicts less IFN-beta expression and potential vulnerability to COVID-19 infection
}

\author{
Amit K. Maiti ${ }^{1}$ (D) \\ Received: 14 May 2020 / Accepted: 23 July 2020 / Published online: 31 July 2020 \\ (C) Springer-Verlag GmbH Germany, part of Springer Nature 2020
}

\begin{abstract}
Covid-19 has caused worldwide devastation. IFIH1 is a pattern recognition receptor that senses coronavirus RNA and triggers interferon production as a first line of viral immune defense. The role of IFIH1 polymorphism, rs 1990760 (C>T; aaA946T) in the epidemiology of viral infection is well studied, and the minor allele T resists viral infection. Knock-in mice with mutated IFIH1 protein $(946 \mathrm{~T})$ for this allele have enhanced interferon production and protection from lethal viral infection. The minor allele frequency (Tmaf) varies widely from Africans $(0.06$ to 0.35$)$ to Chinese $(0.19$ to 0.23$)$ to Caucasians $(0.56$ to 0.69$)$. During the initial days of infection when the social restrictions were not imposed, I show that the infection rate in Italy was lower as expected from its higher Tmaf (0.56) than that in China (Tmaf for southern China, 0.23). The infection rate in the USA and Spain was intermediate between those two countries despite higher Caucasian overall Tmaf (0.69), perhaps due to a more admixed African population in these countries. These analyses suggest that African-Americans and Chinese with low Tmaf of rs1990760 are more vulnerable to SARS-COV2 infection, apart from other genetic factors or socioeconomic conditions in these population. Taken together, an IFN-beta supplement might aid in preventing COVID-19 infection and help in development of herd immunity.
\end{abstract}

Keywords SARS-COV2 $\cdot$ COVID-19 $\cdot$ IFIH1 $\cdot$ Polymorphism $\cdot$ Interferon-beta

Recently, novel coronavirus (virus, SARS-COV2; disease, COVID-19) infection and its related mortality are so severe internationally that the WHO declared it as a pandemic. Although mortality rates differ in various countries, such as in Wuhan (little more than $3 \%$ ) but in Italy, it was so far more than $5 \%$ of infected people. The principle mode of transmission of SARS-COV2 is air-droplet-borne, and eventually, the virus enters lung alveolar cells through the upper respiratory tract to multiply and cause disease. SARS-COV2 has a protein coat with spikes and a positive $\sim 30-\mathrm{kb}(29,903$ base) RNA strand (Ren et al. 2020). After attaching to the host cells with its spikes, it uses the ACE2 receptor as well as the TMPRSS2 enzyme to enter into host cells and uses its host machinery to replicate its RNA genome to make thousands of RNA molecules (Hoffmann et al. 2020). It also uses host protein synthesis system to synthesize its coat proteins to pack its RNA

Amit K. Maiti

akmit123@yahoo.com; amit.maiti@mydnavar.com

1 Department of Genetics and Genomics, Mydnavar, 2645 Somerset Boulevard, Troy, MI 48084, USA genetic material to become a new full-fledged virus and bursts the host cells to come out to infect other cells. Lung cells damaged or destroyed by infection cannot carry out their function of supplying oxygen to the blood (Chan et al. 2020).

The first line of response of host defense system against viral attack is to sense the pathogen via Pattern Recognition Receptors (PRR). One major PRR that first senses the presence of viral RNA is IFIH1 (InterFeron-Induced Helicase 1; MDA5) (Barral et al. 2009). Along with RIG1, IFIH1 is a viral RNA sensor protein but mechanistically differs from RIG-1 as it mainly senses coronavirus, picornavirus, and rhinovirus, whereas RIG1 senses influenza $\mathrm{B}$ and dengue virus (Chistiakov 2010; Loo et al. 2008). IFIH1 induces interferon (IFN) production in the host body to trigger the cellmediated, humoral (antibody production) immunity and also activates MAVS (mitochondrial antiviral system) as host immune response (Belgnaoui et al. 2011). IFIH1 plays an important role in resisting respiratory viral infection in children (Asgari et al. 2017).

The role of IFIH1 sensing and defense has already been demonstrated against another coronavirus, MERS-COV that created Middle East Respiratory Syndrome (MERS). MERS- 
COV Orf4a protein acts as a type 1 IFN antagonist and Orf8b reduces $80 \%$ of the IFIH-mediated IFN-beta production in human cells in the cell culture (Lee et al. 2019; Niemeyer et al. 2013). However, protein sequence alignment of MERS-COV Orf4a and Orf8b with SARS-COV2 proteins does not yield any similarities implying that SARS-COV2 does not possess an orthologue of these two genes. No study has reported about the role of IFIH1 in SARS-COV infection with which SARS-COV2 shares the highest sequence identity (79.8\%) (Ren et al. 2020). Given the role of IFIH1 sensing coronavirus RNA, it is expected that similar phenomena should also prevail for SARS-COV2 as both viruses use ACE2 and TMPRSS2 for host cell entry (Hoffmann et al. 2020), and thus, IFIH1 sensing could be a first line of defense.

Naturally occurring polymorphisms of IFIH1 gene have been widely studied in the population. Among them, rs1990760 (C>T, aaA946T) is associated with several autoimmune diseases (ADs), such as type 1 diabetes (Nejentsev et al. 2009), lupus erythematosus (Molineros et al. 2013), and vitiligo (Jin et al. 2012). We also observed that IFIH1 maintains a feedback loop with IFN production, and upon viral infection, this $(\mathrm{C}>\mathrm{T})$ transition increases IFN-beta production in IFIH1 $946 \mathrm{~T}$ overexpressed cells that in turn increases the expression of other two antiviral genes, IFIT1 and MX1 (Molineros et al. 2013). Recently, the 946T IFIH1-mutated protein was demonstrated to increase IFN-beta production and trigger the MAVS pathway to protect against lethal picornavirus infection in both cell culture and a 946T knock-in mouse model (Gorman et al. 2017). As increased IFN level in the body is a prerequisite for developing some ADs (Baechler et al. 2006), the authors suggested that $946 \mathrm{~T}$ protects hosts from viral infection at the cost of higher risk of some ADs. However, ADs are complex diseases and involve interaction of genetic mutation with environmental factors that remain to be explained. Moreover, in most cases, a single polymorphism increases the risk of developing ADs but symptoms do not develop (Gregersen and Olsson 2009). rs1990760 minor allele T was earlier shown to play important role in resisting viral infection in the population (Fumagalli et al. 2010). From the mouse and cell culture studies, it is expected that the $\mathrm{T}$ allele (946T)-carrying people should have higher IFN levels and a lower risk of SARS-COV2 infection, and thus should be protected from infection. This $\mathrm{C}>\mathrm{T}$ is highly polymorphic in various ethnic populations (Table 1), and if this $\mathrm{T}$ allele has a role in resisting SARS-COV2 infection, we expect a much lower rate of infection in Italian population (mainly Caucasian, Tmaf, 0.56; heterozygosity, 0.58) than Chinese population (Southern Chinese, Tmaf, 0.23; heterozygosity, 0.35). Here I emphasize the rate of infection instead of the total number of infections by expecting a higher number of
Table 1 The MAF of rs1990760 in 1000 genome (https://www. ensembl.org)

\begin{tabular}{|c|c|c|c|}
\hline Population & Cmaf & Tmaf & $\mathrm{C} / \mathrm{T}$ (heterozygosity) \\
\hline AFR (overall) & 0.64 & 0.35 & 0.375 \\
\hline ACB (Caribbean) & 0.78 & 0.22 & 0.188 \\
\hline GWD (Gambia) & 0.79 & 0.21 & 0.27 \\
\hline LWK (Kenya) & 0.89 & 0.10 & 0.182 \\
\hline YRI (Nigeria) & 0.94 & 0.06 & 0.12 \\
\hline AMR (overall) & 0.61 & 0.39 & 0.47 \\
\hline AFR-AMR* & 0.815 & 0.185 & 0.293 \\
\hline EUR-AMR* & 0.397 & 0.603 & 0.469 \\
\hline MXL (Mexico) & 0.56 & 0.43 & 0.53 \\
\hline PEL (Peru) & 0.76 & 0.24 & 0.435 \\
\hline EAS (overall) & 0.81 & 0.19 & 0.30 \\
\hline CHB (Han Chinese) & 0.846 & 0.164 & 0.29 \\
\hline CHS (Southern Chinese) & 0.767 & 0.233 & 0.35 \\
\hline JPT (Japan) & 0.74 & 0.26 & 0.40 \\
\hline EUR (overall) & 0.395 & 0.695 & 0.54 \\
\hline CEU (Caucasian) & 0.384 & 0.616 & 0.545 \\
\hline GBR (Great Britain) & 0.385 & 0.615 & 0.46 \\
\hline IBS (Spain) & 0.355 & 0.645 & 0.449 \\
\hline TSI (Italy) & 0.44 & 0.56 & 0.58 \\
\hline $\mathrm{SAS}^{\$}$ (overall) & 0.436 & 0.564 & 0.50 \\
\hline BEB (Bengali) & 0.494 & 0.506 & 0.523 \\
\hline GIH (Gujrati) & 0.374 & 0.626 & 0.495 \\
\hline ITU (Telegu) & 0.426 & 0.574 & 0.52 \\
\hline STU (Tamil) & 0.47 & 0.53 & 0.52 \\
\hline PJL (Punjabi) & 0.42 & 0.58 & 0.44 \\
\hline
\end{tabular}

*NHLBI Exome Project

${ }^{\$}$ South Asian, Cmaf-major allele frequency of C allele, Tmaf-minor allele frequency of $\mathrm{T}$ allele

protective CT or TT genotype-carrying individuals in a population that will decrease the infection rate with SARS-COV2 than the vulnerable-risk groups with majority of unprotective CC genotype (absence of $\mathrm{T}$ allele)carrying individuals.

We obtained numbers of infections from day-to-day reporting of country-specific infection data at the www. worldometers/info/coronavirus website, which is considered the best free-reference library by the American Library Association (Worldometers 2020). These numbers were used to make bar charts and graphs. In Wuhan, China, and Lombardy, Italy, in the first 12 days where the infection was mostly spontaneous and the social constraints (isolation, quarantine, state-imposed travel ban, and city lockdown) were initially not imposed, the rate of infection was indeed lower in Italy (10149 cases from 889 cases) than China (20440 cases from 830 cases) (Fig. 1a, b, e). 


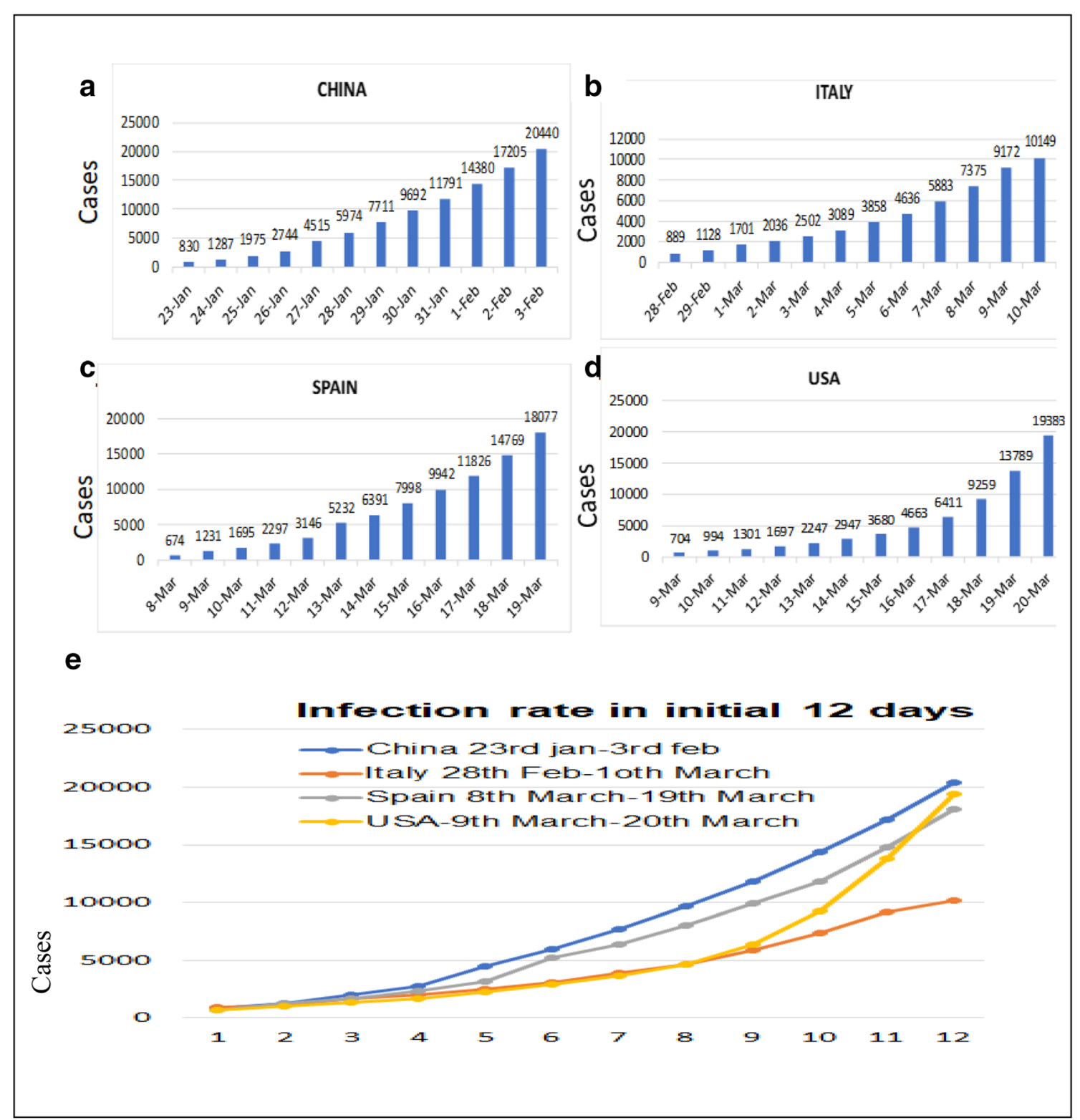

Fig. 1 Infection rate of SARS-COV2 in various countries in initial 12 days of reported infection. All data were obtained from country-specific infection data at https://www.worldometers.info/coronavirus. The numbers obtained from day-to day reporting were used to make bar charts and graphs. a China. b Italy. c Spain. d USA. e Infection rates of all four countries are shown. In a similar timespan of 12 days, infection of cases is doubled in China than that in italy
However, the infection rate with a similar timespan of initial 12 days in Spain (from 674 cases to 18077 cases) and the USA (from 704 cases to 19383 cases) seems higher (Fig. 1c, d, e) than in Italy but lower than in China. Such a high rate of infection in these two countries as compared with that in Italy is not expected from allele frequency of Caucasians (overall Tmaf, 0.69). This may be attributed to the fact that the overall population of these two countries is highly admixed with an African population where the $\mathrm{T}$ allele frequency is very low (Tmaf of Africans overall is 0.35 but ranging from 0.06 to 0.22 ). We also note that in initial days, the infection rates for Italy and the USA are almost the same with a sharp increase of
US infection rate in later days. It could be attributed to initial US data that were obtained mainly from Washington state (Seattle, site of infection, 69\% white and 7.9\% AfricanAmerican population); whereas in later days, it was mainly from New York City (44\% white and $25.5 \%$ AfricanAmerican population) (US States Census Bureau, 2014, 2017).

In these analyses, I did not include data after 12 days of infection because the infection rate was modified and did not proceed spontaneously due to state- or country-imposed restrictions (lockdown etc.). In addition, the severity of death rate could also be dependent on this SNP as the protective T 
allele is responsible for IFN-beta production but currently it is not possible to analyze with limited available data. Also, the mortality rate in various countries depends on many factors, such as an aging population and the health conditions of patients when reported. The limitations of this study include the day-to-day availability of correct number of reported cases and testing abilities that varies in different countries. However, the underreporting of positive cases, most expected in this case, could make the curve of China sharper, emphasizing a higher increase of infection rate than that observed here. Nevertheless, testing and reporting of positive cases were not genotype-specific. It is also to be noted that this SNP or IFIH1 gene may not be the only genetic factor for such vulnerability of infection for African-American population. Again, most of the time, genotype-phenotype relationship also depends on various socioeconomic factors that are mostly prevalent in this population and identifying each of them is obviously of utmost importance.

IFIH1 polymorphisms in various populations were implicated in the population migration upon viral attack from ancestral populations originating from Africa to Europe and Asia, in which the viral-resistance $\mathrm{T}$ allele was directionally selected (Fumagalli et al. 2010). Particularly, the rs 1990760 T allele that also confers susceptibility to ADs originated and was selected after migration as a neutral or mildly deleterious variant in the European and Asian population. The early-age nonindustrial environmental condition in these countries did not allow enough time to develop susceptibility to ADs (Sironi and Clerici 2010).

These large-scale epidemiological data indicate that the role of rs1990760 (A946T) of IFIH1 in SARS-COV2 infection and $\mathrm{T}$ allele-carrying individuals may be more resistant to SARS-COV2 infection. It is also expected that Africans or African-Americans having exceptionally low Tmaf (ranging from 0.06 to 0.35 ) are more vulnerable-risk groups to SARSCOV2 infection than Caucasians and residents of the Indian subcontinent (overall Tmaf, 0.56).

Further investigation with proper large-scale genotyping of affected individuals should be initiated. Nevertheless, initiation of IFN-beta supplement to develop herd immunity and potentially cure SARS-COV2 infected individuals should be considered. IFN-beta is already available and being used as a drug for treatment of multiple sclerosis (MS) (Trinschek et al. 2015). A phase 2 clinical trial in Hong Kong with interferonbeta- $1 \mathrm{~b}$ and proteases, lopinavir-ritonavir, and ribavirin has been reported demonstrating that all mild and moderately affected patients were cured ( $n=86$, with $100 \%$ success rate). They concluded that this triple drug treatment has a superior effect in curing COVID-19 patients than lopinavir-ritonavir or ribavirin alone (Hung et al. 2020). However, they did not include severely affected patients perhaps due to a lack of patients (only 7 deaths until 5 July 2020, www. worldometers.info/coronavirus). Thus, clinical trials of IFN- beta supplement could be considered an effective treatment for COVID-19.

Acknowledgments I thank anonymous reviewers for their valuable comments and advice to improve the quality of this manuscript.

\section{Compliance with ethical standards}

Conflict of interest The author declares that he has no conflict of interest.

\section{References}

Asgari S et al (2017) Severe viral respiratory infections in children with. Proc Natl Acad Sci U S A 114:8342-8347. https://doi.org/10.1073/ pnas. 1704259114

Baechler EC, Batliwalla FM, Reed AM, Peterson EJ, Gaffney PM, Moser KL, Gregersen PK, Behrens TW (2006) Gene expression profiling in human autoimmunity. Immunol Rev 210:120-137. https://doi. org/10.1111/j.0105-2896.2006.00367.x

Barral PM, Sarkar D, Su ZZ, Barber GN, DeSalle R, Racaniello VR, Fisher PB (2009) Functions of the cytoplasmic RNA sensors RIGI and MDA-5: key regulators of innate immunity. Pharmacol Ther 124:219-234. https://doi.org/10.1016/j.pharmthera.2009.06.012

Belgnaoui SM, Paz S, Hiscott J (2011) Orchestrating the interferon antiviral response through the mitochondrial antiviral signaling (MAVS) adapter. Curr Opin Immunol 23:564-572. https://doi.org/ 10.1016/j.coi.2011.08.001

Chan JF et al (2020) A familial cluster of pneumonia associated with the 2019 novel coronavirus indicating person-to-person transmission: a study of a family cluster. Lancet 395:514-523. https://doi.org/10. 1016/S0140-6736(20)30154-9

Chistiakov DA (2010) Interferon induced with helicase C domain 1 (IFIH1) and virus-induced autoimmunity: a review. Viral Immunol 23:3-15. https://doi.org/10.1089/vim.2009.0071

Fumagalli $\mathrm{M}$ et al (2010) Population genetics of IFIH1: ancient population structure, local selection, and implications for susceptibility to type 1 diabetes. Mol Biol Evol 27:2555-2566. https://doi.org/10. 1093/molbev/msq141

Gorman JA et al (2017) The A946T variant of the RNA sensor IFIH1 mediates an interferon program that limits viral infection but increases the risk for autoimmunity. Nat Immunol 18:744-752. https://doi.org/10.1038/ni.3766

Gregersen PK, Olsson LM (2009) Recent advances in the genetics of autoimmune disease. Annu Rev Immunol 27:363-391. https://doi. org/10.1146/annurev.immunol.021908.132653

Hoffmann M et al. (2020) SARS-CoV-2 Cell Entry Depends on ACE2 and TMPRSS2 and Is Blocked by a Clinically Proven Protease Inhibitor. Cell doi:https://doi.org/10.1016/j.cell.2020.02.052

Hung et al (2020) Triple combination of interferon beta-1b, lopinavirritonavir, and ribavirin in the treatment of patients admitted to hospital with COVID-19: an open label, randomized, phase 2 trial. The Lancet 395:1695-1704. https://doi.org/10.1016/S0140-6736(20) 31042-4

Jin $\mathrm{Y}$ et al (2012) Genome-wide association analyses identify 13 new susceptibility loci for generalized vitiligo. Nat Genet 44:676-680. https://doi.org/10.1038/ng.2272

Lee JY, Bae S, Myoung J (2019) Middle East respiratory syndrome coronavirus-encoded ORF8b strongly antagonizes IFN- $\beta$ promoter activation: its implication for vaccine design. J Microbiol 57:803811. https://doi.org/10.1007/s12275-019-9272-7 
Loo YM et al (2008) Distinct RIG-I and MDA5 signaling by RNA viruses in innate immunity. J Virol 82:335-345. https://doi.org/10. 1128/JVI.01080-07

Molineros J et al (2013) Admixture mapping in lupus identifies multiple functional variants within IFIH1 associated with apoptosis, inflammation and autoantibody production. PLOS Genetics 9(2): e1003222. https://doi.org/10.1371/journal.pgen.1003222

Nejentsev S, Walker N, Riches D, Egholm M, Todd JA (2009) Rare variants of IFIH1, a gene implicated in antiviral responses, protect against type 1 diabetes. Science 324:387-389. https://doi.org/10. 1126/science. 1167728

Niemeyer D et al (2013) Middle East respiratory syndrome coronavirus accessory protein $4 \mathrm{a}$ is a type I interferon antagonist. J Virol 87: 12489-12495. https://doi.org/10.1128/JVI.01845-13

Ren LL et al. (2020) Identification of a novel coronavirus causing severe pneumonia in human: a descriptive study. Chin Med J (Engl) doi: https://doi.org/10.1097/CM9.0000000000000722

Sironi M, Clerici M (2010) The hygiene hypothesis: an evolutionary perspective. Microbes Infect 12:421-427. https://doi.org/10.1016/j. micinf.2010.02.002
Trinschek B, Luessi F, Gross CC, Wiendl H, Jonuleit H (2015) Interferon-beta therapy of multiple sclerosis patients improves the responsiveness of $\mathrm{T}$ cells for immune suppression by regulatory. $\mathrm{T}$ cells Int J Mol Sci 16:16330-16346. https://doi.org/10.3390/ ijms160716330

US Census Bureau (2014) "Seattle (City), Washington". State \& County QuickFacts. U.S. Census Bureau. A Archived from the original on March 27, 2014. Retrieved April 26, 2014.

US Census Bureau (2017) QuickFacts for New York City/New York State/United States, United States Census Bureau. Retrieved February 9, 2017.

Worldometers (2020) Real time statistics | Blog | National Library of New Zealand. natlib.govt.nz.

Publisher's note Springer Nature remains neutral with regard to jurisdictional claims in published maps and institutional affiliations. 